\title{
A REMARK ON BONY MAXIMUM PRINCIPLE
}

\author{
P. L. LIONS
}

\begin{abstract}
We extend a result due to J. M. Bony concerning a form of the classical maximum principle adapted to Sobolev spaces. We treat the case of the limiting exponent and show that the result is optimal. We give various applications to nonlinear elliptic partial differential equations.
\end{abstract}

I. Introduction. In [8] J. M. Bony gave a new form of the classical maximum principle adapted to Sobolev spaces. Our purpose here is to present a slight extension of [8] that we prove to be optimal.

More precisely, in [8] is proved the following result: let $\Omega$ be an open set in $R^{n}$ and let $u \in W_{\text {loc }}^{2, p}(\Omega)=\left\{u, D^{\alpha} u \in L_{\text {loc }}^{p}(\Omega)\right.$ for $\left.|\alpha| \leqslant 2\right\}$. Then if $x_{0}$ is a point of local maximum of $u$ and if $p>n$, we have

$$
\liminf _{y \rightarrow x_{0}} \operatorname{ess} \sum_{i, j} a_{i j}(y) \partial_{i j} u(y) \leqslant 0
$$

where $\left(a_{i j}\right)_{1 \leqslant i, j \leqslant n}$ is nonnegative a.e. in $\Omega$ and $a_{i j} \in L_{\text {loc }}^{\infty}(\Omega)$.

This result has proved to be very useful for uniqueness and comparison results (see for example [8], H. Amann and M. G. Crandall [7], P. L. Lions [19] and L. C. Evans [13]) concerning various linear or nonlinear second-order elliptic equations.

We show here that (1) still holds if $p=n$ and more precisely that we have if $p=n$

$$
\limsup _{y \rightarrow x_{0}} \operatorname{ess} \bar{\lambda}(y) \leqslant 0, \quad \liminf _{y \rightarrow x_{0}} \operatorname{ess}|D u(y)|=0
$$

where $\bar{\lambda}(y)$ is the highest eigenvalue of $\left(D^{2} u(y)\right)$. It is worth pointing out that when $p>n$ then (1) and $\left(1^{\prime}\right)$ are equivalent since by Sobolev imbeddings $u \in C^{1}(\Omega)$ and $D u\left(x_{0}\right)=0$.

Actually we give below ( $(I I)$ a result even more precise than $\left(1^{\prime}\right)$; and we show that $p=n$ is the limiting case in a very sharp way since we give below an example when $u \in W_{\text {loc }}^{2, p}(\Omega)$ for all $p<n$ and such that $D^{2} u \in M_{\text {loc }}^{n}(\Omega)$ (weak- $L^{n}$ space) but nevertheless $\left(1^{\prime}\right)$ does not hold.

Of course this extension has the same applications as those mentioned above but we will skip them. Instead we apply this result in §III to show some properties of solutions of nonlinear elliptic equations.

At this point we want to mention the pioneering study on the maximum principle by A. D. Alexandrov [1-5] and the fact that our proof is based in an essential way

Received by the editors August 9, 1982.

1980 Mathematics Subject Classification. Primary 35J15, 35J65.

Key words and phrases. Sobolev spaces, maximum principle, second-order elliptic equations, viscosity solutions. 
on a result taken from [1] (that we reprove by a different method in the appendix for the reader's convenience). Let us also mention that our argument will use some ideas due to C. Pucci and G. Talenti [27] and M. G. Crandall and P. L. Lions [11]. Finally we would like to point out that some of the uniqueness results proved in [8, 7 and 19] (for example) using Bony maximum principle can thus, as we said before, be extended to $W_{\text {loc }}^{2, n}(\Omega)$ solutions but that alternate (and related) proofs can be made using probabilistic arguments due to N. V. Krylov [15] and in particular a powerful inequality $[16,17]$ which in some sense is the probabilistic counterpart of $A$. D. Alexandrov estimates [2,3]-see also C. Pucci [26].

II. The main result. Let $\Omega$ be an open set in $R^{n}$. If $x_{0} \in \Omega$, we denote by $B\left(x_{0}, \delta\right)$ the open ball of center $x_{0}$ and radius $\delta$.

Proposition 1. Let $u \in W_{\mathrm{loc}}^{2, n}(\Omega)$ and assume that $u$ has a local strict maximum at some point $x_{0} \in \Omega$. Then there exists $\varepsilon \in C\left(R_{+}, R_{+}\right)$satisfying $\lim _{t \rightarrow 0+} \varepsilon(t)=0$ such that for all $\delta>0$ small enough the set $A_{\delta}=\left\{y \in B\left(x_{0}, \delta\right),\left(D^{2} u(y)\right) \leqslant 0, u\right.$ is differentiable at $y$ and $|D u(y)| \leqslant \varepsilon(\delta)\}$ has a positive measure.

This result immediately implies the following

Corollary 2. Let $u \in W_{\mathrm{loc}}^{2, n}(\Omega)$ and let $x_{0} \in \Omega$ be a point of local maximum of $u$. Then (1') holds.

Proof of Corollary 2. Clearly $v(x)=u(x)-\left|x-x_{0}\right|^{4}$ has a local strict maximum at $x=x_{0}$, and we conclude by applying Proposition 1 to $v$.

EXAMPLE. We give now a very simple example showing that the above result is, in some sense, sharp: take $\Omega=R^{n}$ with $n \geqslant 2$ and let $u(x)=-|x|$. Obviously $u \in$ $W_{\mathrm{loc}}^{2, p}(\Omega)$ for all $p<n$ and $D^{2} u \in M^{n}(\Omega)$. In addition $u$ has a global strict maximum at 0 ; nevertheless for all $\delta>0$ such that $\varepsilon(\delta)<1$ the set $A_{\delta}$ is empty!

Proof of Proposition 1. The idea of the proof consists in looking at "the points of concavity of $u$ " in $B\left(x_{0}, \delta\right)$ and then proving that this set (essentially $A_{\delta}$ ) has a positive measure.

First, following C. Pucci and G. Talenti [27] we claim that for all $\delta>0$ (such that $\left.\overline{B\left(x_{0}, \delta\right)} \subset\left\{y \in \Omega / u(y)<u\left(x_{0}\right)\right\}\right)$ and for $\xi \in B(0, \varepsilon)$ with

$$
\varepsilon=\varepsilon(\delta)=\frac{1}{\delta}\left\{u\left(x_{0}\right)-\sup _{\partial B\left(x_{0}, \delta\right)} u\right\},
$$

there exists $y \in B\left(x_{0}, \delta\right)$ such that

$$
u(x)+(\xi, x) \leqslant u(y)+(\xi, y) \text { for all } x \in \overline{B\left(x_{0}, \delta\right)} .
$$

Indeed from the choice of $\varepsilon$ we have $u\left(x_{0}\right)+\left(\xi, x_{0}\right)>\sup _{\partial B\left(x_{0}, \delta\right)}\{u(y)+(\xi, y)\}$, and this implies the existence of $y$.

Let us point out that without loss of generality we may assume $\varepsilon(\delta) \rightarrow 0$ as $\delta \rightarrow 0+$ (or replace $\varepsilon(\delta)$ by $\varepsilon(\delta) \wedge \delta$ ).

We then set $A_{\delta}^{\prime}=\left\{y \in B\left(x_{0}, \delta\right) / \exists \xi \in B(0, \varepsilon)\right.$ such that $u(x)+(\xi, x) \leqslant u(y)+$ $\left.(\xi, y), \forall x \in \overline{B\left(x_{0}, \delta\right)}\right\}$. By a classical result of A. D. Alexandrov [1] (part V)-that we prove by a different method in the appendix-we deduce $A_{\delta}^{\prime}$ has a positive 
measure. Actually (see the proof in the appendix) one has

$$
\int_{A_{\delta}^{\prime}}\left\{\operatorname{det}\left(-D^{2} u\right)\right\}^{+} d x \geqslant c_{n} \varepsilon(\delta)^{n}
$$

where $c_{n}$ denotes the volume of $B(0,1)$.

Since by well-known results on Sobolev spaces $u$ is a.e. differentiable and since if $y \in A_{\delta}^{\prime}$ and if $u$ is differentiable at $y$ then $\xi=D u(y)$ we see that

$$
\operatorname{meas}\left(A_{\delta}^{\prime}-A_{\delta}^{\prime \prime}\right)=0
$$

where $A_{\delta}^{\prime \prime}=\left\{y \in A_{\delta}^{\prime}, u\right.$ is differentiable at $\left.y\right\}$.

We conclude by proving that a.e. on $A_{\delta}^{\prime \prime}$ we have $\left(D^{2} u\right) \leqslant 0$. Indeed let $\left(\xi_{m}\right)_{m \geqslant 1}$ be a dense sequence in $S^{n-1}$, then except on a set of zero Lebesgue measure we have

$$
\frac{1}{h^{2}}\left\{u\left(x+h \xi_{m}\right)+u\left(x-h \xi_{m}\right)-2 u(x)\right\} \underset{h \rightarrow 0+}{\rightarrow} \frac{\partial^{2} u}{\partial \xi_{m}^{2}}, \quad \forall m \geqslant 1
$$

Therefore a.e. on $A_{\delta}^{\prime \prime}$ we deduce $\partial^{2} u / \partial \xi_{m}^{2} \leqslant 0, \forall m \geqslant 1$; thus $\left(D^{2} u\right) \leqslant 0$, and we conclude.

REMARKs. (i) The above proof shows that we have

$$
\int_{A_{\delta}} \operatorname{det}\left(-D^{2} u\right) d x \geqslant c_{n} \varepsilon(\delta)^{n}, \quad \varepsilon(\delta)=\frac{1}{\delta}\left\{u\left(x_{0}\right)-\sup _{\partial B\left(x_{0}, \delta\right)} u(x)\right\} .
$$

Let us mention that if $u(x)=-\lambda\left|x-x_{0}\right|^{2}$ (for some $\lambda \geqslant 0$ ) then (2') becomes an equality!

(ii) It is clear that the main point in the above proof where we used the fact that $u \in W_{\mathrm{loc}}^{2, n}$ is when we obtained (2). This remark may be used to extend Proposition 1 a little bit to functions $u$ of the form $u=u_{1}+u_{2}$ with $u_{2} \in W_{\text {loc }}^{2, n}(\Omega)$ and $u_{1}$ concave, finite on $\Omega$ and with an absolutely continuous normal image. Various (technical) extensions of this form are possible but we will skip them.

(iii) Let us emphasize that by Corollary 2, we have proved that if $u \in W_{\text {loc }}^{2, n}(\Omega)$ and has a local maximum at $x_{0} \in \Omega$ then

$$
\liminf _{y \rightarrow x_{0}} \operatorname{ess}|D u(y)|=0
$$

and this no longer holds for $u(x)=-\left|x-x_{0}\right|\left(\in W_{\text {loc }}^{2, p}(\Omega)\right.$, for $p<n, D^{2} u \in$ $M^{n}(\Omega)$ ). It is also interesting to point out that in this counterexample $u$ is concave and its normal measure is $\delta_{x_{0}}$.

III. An application of Proposition 1. Let us consider a fully nonlinear second-order elliptic equation of the form

$$
F\left(D^{2} u, D u, u, x\right)=0 \text { in } \Omega
$$

where $F \in C\left(L_{S}^{n} \times R^{n} \times R \times \Omega\right)$ and $L_{S}^{n}=\{A n \times n$ symmetric matrix $\}$. We express the fact that (4) is elliptic (eventually degenerate) by assuming

$$
F(A, p, t, x) \leqslant F(B, p, t, x) \text { if } A \geqslant B, \forall(p, t, x) \in R^{n} \times R \times \Omega .
$$


We recall the notion of viscosity solutions of $(4): u \in C(\Omega)$ is said to be a viscosity solution of (4) if it satisfies for all $\varphi \in C^{2}(\Omega)$ the following conditions:

$$
\begin{gathered}
\left\{\begin{array}{r}
\text { at any local maximum point } x_{0} \text { of }(u-\varphi), \text { one has } \\
F\left(D^{2} \varphi\left(x_{0}\right), D \varphi\left(x_{0}\right), u\left(x_{0}\right), x_{0}\right) \leqslant 0 ;
\end{array}\right. \\
\left\{\begin{array}{c}
\text { at any local minimum point } x_{0} \text { of } u-\varphi, \text { one has } \\
F\left(D^{2} \varphi\left(x_{0}\right), D \varphi\left(x_{0}\right), u\left(x_{0}\right), x_{0}\right)>0 .
\end{array}\right.
\end{gathered}
$$

In P. L. Lions [20, 21] equivalent definitions are given. This notion of solution of (4) has been introduced in the context of first order Hamilton-Jacobi equations (i.e. (4) with $F(A, p, t, x)=F(p, t, x))$ by M. G. Crandall and P. L. Lions [11] (see also $[12,10])$ for uniqueness and stability purposes. Some properties (stability, uniqueness, ...) of these solutions are presented in P. L. Lions $[\mathbf{2 0 , 2 1 , 2 2 ]}$.

Of course we might consider a solution $u \in W_{\text {loc }}^{2, p}(\Omega)$ (for some $1 \leqslant p \leqslant \infty$ ) of (4), i.e.

$$
F\left(D^{2} u, D u, u, x\right)=0 \quad \text { a.e. in } \Omega
$$

and a reasonable question is to decide whether $u$ is a viscosity solution of (4). This is answered by the following

Corollary 3. Let $u \in W_{\text {loc }}^{2, n}(\Omega)$ satisfy $\left(4^{\prime}\right)$; then $u$ is a viscosity solution of (4).

Proof. Let us check for example (6). Obviously $v=u-\varphi \in W_{\text {loc }}^{2, n}(\Omega)$ satisfies

$$
F\left(D^{2} v(x)+D^{2} \varphi(x), D v(x)+D \varphi(x), u(x), x\right)=0 \text { a.e. }
$$

We may then apply Corollary 2 to deduce (6), since $F$ satisfies (5) and $F$ is continuous.

REMARK. The (counter-) example given in \$II shows also that Corollary 3 is, in some sense, optimal since $u(x)=-|x|\left(\in W_{\text {loc }}^{2, p}\left(R^{n}\right)\right.$ for $\left.p<n, D^{2} u \in M^{n}\left(R^{n}\right)\right)$ solves (4') for $F(A, p, t, x)=1-|p|$. But (6) does not hold (take $\varphi=0, x_{0}=0$ ).

Appendix. This section is devoted to the sketch of a new proof of the result of A. D. Alexandrov we used in the proof of Proposition 1. Let $B$ be a ball in $R^{n}$ and let $v \in W^{2, n}(B)$. Let $M$ be a borel set in $B$ and let $N$ be a borel set in $R^{n}$. We assume

$$
\forall \xi \in N, \exists x \in M, v(y) \geqslant v(x)+(\xi, y-x) \text { for } y \in \bar{B}
$$

Then we have

Proposition 4. With the above notations and assumptions, we have

$$
\int_{M}\left\{\operatorname{det}\left(D^{2} v\right)\right\}^{+} d x \geqslant \operatorname{meas}(N)
$$

REMARKs. (i) This result extends slightly A. D. Alexandrov's result in [1] where (8) is proved with $\left|\operatorname{det}\left(D^{2} v\right)\right|$ instead of $\left\{\operatorname{det}\left(D^{2} v\right)\right\}^{+}$.

(ii) In the proof of Proposition 2, we used (8) with $v=-u, N=B(0, \varepsilon(\delta))$ and $M=A_{\delta}^{\prime}$. 
Proof. Let $w$ be the largest convex function on $\bar{B}$ such that $w \leqslant v$ on $\bar{B}$. Clearly $M \subset\{x \in B / v(x)=w(x)\}$ and $N \subset \partial w(M)$ where $\partial w$ is the subdifferential of the convex function $w$. By definition of the normal measure $\mu$ of $w$ (see A. D. Alexandrov [6], A. V. Pogorelov [25] and S. Y. Cheng and S. T. Yau [9]) we see that $\operatorname{meas}(N) \leqslant \mu(M)(=\operatorname{meas}(\partial w(M)))$. Therefore (8) amounts to proving that $\mu$ is absolutely continuous with respect to the Lebesgue measure and that, if we still denote by $\mu$ its density, we have

$$
0 \leqslant \mu \leqslant\left\{\operatorname{det}\left(D^{2} v\right)\right\}^{+} \quad\left(\in L^{1}(B)\right) .
$$

We may then conclude by general results on obstacle problems for Monge-Ampère equations (cf. P. L. Lions [23]). Let us just indicate briefly why ( $\left.8^{\prime}\right)$ holds. It is quite clear that $w$ is the (weak) solution (in the sense of Alexandrov) of the following obstacle problem:

$$
\max \left(-\operatorname{det}\left(D^{2} u^{\cdot}\right), w^{\prime}-v\right)=0 \quad \text { in } \Omega, w^{\text {is convex on } \bar{\Omega} .},=v \text { on } \partial \Omega .
$$

Then, since " $\mu=\operatorname{det}\left(D^{2} w^{\prime}\right) ",\left(8^{\prime}\right)$ appears as a particular case of the celebrated Lewy-Stampacchia inequality for obstacle problems (see H. Lewy and G. Stampacchia [18] and D. Kinderlehrer and G. Stampacchia [14]). This interpretation is completely justified in [23] where, following the method introduced in P. L. Lions $[23,24], w$ is shown to be the limit of $w_{\varepsilon}$ solution of

$$
\left\{\begin{array}{l}
\max \left(-\operatorname{det}\left(D^{2} w_{F}+\frac{1}{\varepsilon} p\left(w_{\varepsilon}-v_{\varepsilon}\right) I_{N}\right), w_{F}-v_{\varepsilon}\right)=0 \quad \text { a.e. in } R^{n}, \\
w_{F} \in W^{2 . \infty}\left(R^{n}\right), \quad\left(D^{2} w_{\varepsilon}+\frac{1}{\varepsilon} p\left(w_{F}-v_{\varepsilon}\right) I_{N}\right) \geqslant 0 \quad \text { a.e. in } R^{n},
\end{array}\right.
$$

where $v_{f} \in C_{b}^{\infty}\left(R^{n}\right), v_{\varepsilon} \rightarrow_{\varepsilon \rightarrow 0} v$ in $W^{2 \cdot n}(B)$ and $p \in C_{b}^{\infty}\left(R^{n}\right)$ satisfies $p \equiv 0$ in $\bar{B}$; $\forall \varepsilon>0, \exists \delta>0, p(x) \geqslant \delta$ if $\operatorname{dist}(x, \bar{B}) \geqslant \varepsilon$. Exactly as in [23, 24], one solves (10) and one proves that $w_{\varepsilon}$ converges uniformly on $\bar{B}$ to $w$. Remarking that on $B$ we have

$$
0 \leqslant \operatorname{det}\left(D^{2} w_{\varepsilon}\right) \leqslant\left\{\operatorname{det}\left(D^{2} v_{\varepsilon}\right)\right\}^{+} \underset{\varepsilon \rightarrow 0}{\rightarrow}\left\{\operatorname{det}\left(D^{2} v\right)\right\}^{+} \text {in } L^{1}(B),
$$

we conclude since $\operatorname{det}\left(D^{2} w_{\varepsilon}\right)$ converges weakly in the sense of measures to $\mu$ (see for example [9]). Details on this proof are given in [23].

\section{REFERENCES}

1. A. D. Alexandrov, Investigations on the maximum principle, Izv. Vysš. Učebn. Zaved Matematika. I, 5 (1958), 126-157; II, 3 (1959), 3-12; III, 5 (1959), 16-32; IV, 3 (1960), 3-15; V, 5 (1960), 16-26; VI, 1 (1961), 3-20. (Russian)

2. Uniqueness conditions and estimates for the solution of the Dirichlet problem, Amer. Math. Soc. Transl. (2) 68 (1968), 89-119.

3. Majorization of solutions of second-order linear equations, Amer. Math. Soc. Transl. (2) 68 (1968), 120-143.

4. Majorants of solutions and uniqueness conditions for elliptic equations, Amer. Math. Soc. Transl. (2) 68 (1968), 144-161.

5. __ The impossibility of general estimates for solutions and of uniqueness conditions for linear equations with norms weaker than in $L_{n}$, Amer. Math. Soc. Transl. (2) 68 (1968), 162-168.

6. Dirichlet's problem for the equation Det $\left\|z_{i j}\right\|=\Phi\left(z_{1}, \ldots, z_{n}, z, x_{1}, \ldots, x_{n}\right)$. I, Vestnik Leningrad Univ. Mat. Meh. Astronom. 13 (1958), 5-24. (Russian) 
7. H. Amann and M. G. Crandall, On some existence theorems for semilinear elliptic equations, Indiana Univ. Math. J. 27 (1978), 779-790.

8. J. M. Bony, Principe du maximum dans les espaces de Sobolev, C. R. Acad. Sci. Paris Ser. A 265 (1967), 333-336.

9. S. Y. Cheng and S. T. Yau, On the regularity of the Monge-Ampere equation $\operatorname{det}\left(\partial^{2} u / \partial x_{i} \partial x_{j}\right)=$ $F(x, u)$, Comm. Pure Appl. Math. 30 (1977), 41-68.

10. M. G. Crandall, L. C. Evans and P. L. Lions, Some properties of viscosity solutions of Hamilton-Jacobi equations, Trans. Amer. Math. Soc. (to appear).

11. M. G. Crandall and P. L. Lions, Viscosity solutions of Hamilton-Jacobi equations, Trans. Amer. Math. Soc. (to appear).

12. Condition d'unicité pour les solutions généralisées des équations de Hamilton-Jacobi du premier ordre, C. R. Acad. Sci. Paris Ser. A 292 (1981), 183-186.

13. L. C. Evans, A convergence theorem for solutions of nonlinear second-order elliptic equations, Indiana Univ. Math. J. 27 (1978), 875-887.

14. D. Kinderlehrer and G. Stampacchia, An introduction to variational inequalities and their applications, Academic Press, New York, 1980.

15. N. V. Krylov, Controlled diffusion processes, Springer-Verlag, Berlin, 1980.

16. An inequality in the theory of stochastic integrals, Theory Probab. Appl. 17 (1972), 114-131.

17. Some estimates of the probahility density of a stochastic integral, Math. USSR-Izv. 8 (1974), 233-254.

18. H. Lewy and G. Stampacchia, On the smoothness of superharmonics which solve a minimum problem. J. Analyse Math. 23 (1970), 227-236.

19. P. L. Lions, Résolution analytique des problemes de Bellman-Dirichlet, Acta Math. 146 (1981), $151-166$.

20. Optimal stochastic control of diffusion type processes and IIamilton-Jacobi-Bellman e'quations. Advances in Filtering and Optimal Stochastic Control (Eds., W. H. Fleming and L. (iorostiza). Springer. Berlin, 1982.

21. Optimal control of diffusion processes and IIamilton-Jacobi-Bellman equations (preprint).

22. Sur les équations de Monge-Ampère. III (in proparation).

23. Une méthode nouvelle pour l'existence de solutions régulières de l'équations de Monge-Ampiere.

C. R. Acad. Sci. Paris Ser. A 293 (1981), 589-592.

24. Sur les équations de Monge-Ampere. I, Manuscripta Math. (to appear); II, Arch. Rational Mech. Anal. (to appear).

25. A. V. Pogorelov, The Minkowski multidimensional problem, Wiley, New York, 1978.

26. C. Pucci, Limitazioni per soluzioni di equazioni ellittiche, Ann. Mat. Pura Appl. 74 (1966), 15-30.

27. C. Pucci and (;. Talenti, Elliptic (second-order) partial differential equations with measurable' coefficients and approximating integral equations, Adv. in Math. 19 (1976), 48-105.

Cfremade, Universite Paris IX - Dauphine, Place de lattre de Tassigny, 75775 Paris Cedex 16. FRANCE 